\title{
Schiff bases of indoline-2,3-dione (isatin) with potential antiproliferative activity
}

\author{
Tarek Aboul-Fadl ${ }^{1,2^{*}}$, Awwad A Radwan ${ }^{2,3}$, Mohamed I Attia ${ }^{1}$, Abdullah Al-Dhfyan ${ }^{4}$ and Hatem A Abdel-Aziz ${ }^{1}$
}

\begin{abstract}
Background: Cancer is one of the most dreaded diseases and it is a leading cause of mankind death worldwide. Recent reports documented a remarkable antiproliferative activity of isatin nucleus against various cancer cell lines. The current work describes the antiproliferative activity of Schiff bases of combinatorial mixtures of the isatin derivatives M1-M22 as well as the individual compounds 1-11(A-K) of these combinatorial mixtures.

Results: The designed combinatorial library composed from eleven hydrazides A-K and eleven isatin derivatives 1-11 has been synthesized to formally generate 22 mixtures, M1-M22 of 121 Schiff bases, and their antiproliferative activity against $\mathrm{K} 562$ chronic myelogenous leukemia cells was evaluated. The indexed method of analysis of the prepared library was applied to elucidate the active components in the tested mixtures M1-M22. The predictions from the crossing procedure was validated through evaluation of the antiproliferative activity of individual compounds 1-11(A-K) of the library. Individual compounds 1-11(A-K) were also evaluated against the non-tumorigenic MCF-12A cell line to investigate their selectivity. A pharmacophore model was developed to further optimize the antiproliferative activity among this series of compounds.

Conclusions: Variable antiproliferative activity was revealed with the investigated mixtures M1-M22 and the individual compounds 1-11(A-K). Most of the tested mixtures and several individual Schiff bases displayed high potency with $\mathrm{IC}_{50}$ values in the low micromolar range. A considerable selectivity of some individual compounds to the tumorigenic $\mathrm{K} 562$ cell line compared with the non-tumorigenic MCF-12A cell line was observed as indicated by their selectivity index (SI).
\end{abstract}

Keywords: Isatin, Schiff bases, Combinatorial library, Antiproliferative, Pharmacophore

\section{Background}

Cancer is one of the most dreaded diseases of mankind. It is a leading cause of death throughout the world, and currently, one in 4 deaths in the United States is due to cancer [1]. More than ten million new cancer cases occur annually, roughly half of which is in the developed countries, and the disease causes over six million deaths a year $[2,3]$. Unlimited and uncontrolled cell proliferation is obviously characteristics of tumor cells [4].

Despite several decades of intensive research, the longterm outlook for patients with aggressive cancer remains discouraging, and there is a need for innovative approaches to design anticancer drugs with reduced

\footnotetext{
* Correspondence: fadl@ksu.edu.sa

'Department of Pharmaceutical Chemistry, College of Pharmacy, King Saud University, P.O. Box 2457, Riyadh 11451, Saudi Arabia

${ }^{2}$ Faculty of Pharmacy, Assiut University, Assiut 71526, Egypt

Full list of author information is available at the end of the article
}

toxicity and improved therapeutic indices [5,6]. In recent years, compounds containing hydrazide or hydrazone moieties are attractive target compounds for new drug development because of their potentially versatile biological activities involving antiproliferative activities $[7,8]$. Several studies have been devoted to the antiproliferative activity of aroylhydrazone derivatives [7,9-12]. It was suggested that the antiproliferative activity of these hydrazones may be attributed to inhibition of kinases [13-15], or through generation of radicals and dissipation of the mitochondrial membrane potential [16].

Furthermore, there are significant reasons for investigating the antiproliferative activity of Schiff base derivatives of indolin-2,3-dione (isatin) as recent reports documented a remarkable antiproliferative activity of isatin nucleus against various cancer cell lines [17-22]. Isatins have multiple molecular mechanisms to exert their anticancer activity. Among which tyrosine kinase
(C) Chemistry Central

C 2012 Aboul-Fadl et al.; licensee Chemistry Central Ltd. This is an Open Access article distributed under the terms of the Creative Commons Attribution License (http://creativecommons.org/licenses/by/2.0), which permits unrestricted use, distribution, and reproduction in any medium, provided the original work is properly cited. 
Inhibiton (TKIs), inhibition of cyclin-dependent kinases (CDKs) by binding to the ATP pocket and/or caspase inhibition [23-25].

In continuation to our synthetic work on Schiff bases of isatin with potential biological activity [26-32], the current work describes the antiproliferative activity of Schiff bases of combinatorial mixtures of the isatin derivatives M1-M22 as well as the individual compounds 1-11(A-K) of these combinatorial mixtures. Moreover, Ligand based pharmacophore modeling of these Schiff bases was conducted to evaluate the common features essential for activity and the hypothetical geometries adopted by these ligands in their most active conformers were undertaken.

\section{Results and discussion}

The designed combinatorial library mixtures M1-M22 of the Schiff bases as well as its individual compounds A-K(1-11) were already published $[27,29,30]$. They were obtained either by conventional or microwave assisted methods and are summarized in Table 1.

In vitro antiproliferative activity of the combinatorial mixtures M1-M22 was carried out by use of WST-1 reagent for determination of $\mathrm{IC}_{50}$ for each mixture against K562 chronic myelogenous leukemia cells according to the protocol mentioned in the experimental section and results are given in Table 1 . Variable antiproliferative activity was observed with the investigated mixtures in the following decreasing order: M6 $>$ M22 $>$ M5 $>$ M15 $>$ M7 $>$ M4 $>$ M1 $>$ M3 $>$ M9 $>$ M18 $>$ M2 $=$ M10 $>$ M16 $>$ M11 $>$ M19 $>$ M14 with $\mathrm{IC}_{50}$ range values from 4.48 to $22.6 \mu \mathrm{M}$. Other mixtures did not show significant antiproliferative activity against MCF-12A cell line $\left(\mathrm{IC}_{50}>100 \mu \mathrm{M}\right)$. The most active mixtures in the first set are mixtures M5-M7 whereas in the second set are mixtures M15, M18, and M22. The indexed method of analysis of the prepared library was applied to elucidate the active components in the tested mixtures M1-M22. Intersection of the active rows M1-M7, and M9-M11 with the active columns M12, M14-M16, M18, M19, and M22 gave the location of the possible active components in these mixtures, distinguished by bold cell borders (see Additional file 1).

In order to confirm the reliability of the predictions from the crossing procedure, the synthesized individual compounds A-K(1-11) were also investigated against K562 cell line. From the scattered data given in Table 2, it was difficult to determine precisely the essential moieties in compounds $\mathbf{1 - 1 1}(\mathbf{A}-\mathbf{K})$ required to elicit antiproliferative activity. A general conclusion, however, can be made about the SAR in the synthesized series of hydrazones 1-11(A-K) that the integrated molecular structure features are responsible for the elucidated antiproliferative activity irrespective of the building blocks incorporated in individual molecules. According to the displayed antiproliferative activity of the title compounds

1-11(A-K) they can be divided into: highly active candidates with $\mathrm{IC}_{50}<10 \mu \mathrm{M}$ (B5, D2-D11, E1-E4, E6, E7, E9, E10, F1, F8-F11, G1-G7, G10, H2, H5, H6, H11, I2, I6, J5, and J6), moderately active candidates with $\mathrm{IC}_{50}<20 \mu \mathrm{M}$ (B3, B9, B10, C1, C3, D1, D3, E5, E8, E11, F2-F7, G8, H1, I1, I3, I4, I10, J4, and J7), weekly active with $\mathrm{IC}_{50} \sim 20<100 \mu \mathrm{M}$ (A1-A4, A9, B1, B2, B4, B7, B8, B11, C2, C4, C5, H4, H7-H10, I5, I9, J2, J8, J9, and K10) and inactive with $\mathrm{IC}_{50}>100 \mu \mathrm{M}$ (rest of the compounds). It is clear that series A with isonicotinic acid hydrazide, $\mathbf{B}$ with nicotinic acid hydrazide, $\mathbf{C}$ with furan-2-carboxylic acid hydrazide and $\mathbf{K}$ with nalidixic acid hydrazide have the least contribution in antiproliferative activity.

Surprisingly, antiproliferative activity prediction of the individual compounds from the intersection of M1-M11 and M12-M22 was not consistent with the results achieved from practical investigations of individual compounds particularly in case of $\mathbf{A}$ and $\mathbf{K}$ series. This may be attributed to the additive contribution of the active components in their mixture that are effective than that tested separately.

To evaluate the selectivity of these individual compounds on the tumorigenic cells, their cytotoxicity was measured by cell growth inhibition assay against MCF12A cell line. The MCF-12A cell line is a non-tumorigenic epithelial cell line established from tissue taken at reduction mammoplasty from a nulliparous patient with fibrocystic breast disease that contained focal areas of intraductal hyperplasia. The general in vitro cytotoxic evaluation of these synthesized compounds was carried out also by use of WST-1 reagent for determination of $\mathrm{IC}_{50}$ for each compound according to the protocol mentioned in the experimental section and results are given in Table 2. The selectivity index (SI) which represents $\mathrm{IC}_{50}$ for normal cell line/ $\mathrm{IC}_{50}$ for cancerous cell line. As the SI demonstrates the differential activity of a compound, the greater the SI value is, the more selective it is. Variable selectivity was observed with the different investigated compounds (Table 2). Though it is difficult to contribute the selectivity pattern to either of the building blocks, the E series with benzofuran moiety revealed good selectivity pattern. Among the highly active candidates, compounds D2, D10, D11, F9, F10, F11, G3-G7, G10, H2, H5, J5, and $\mathbf{J 6}$ were the least selective but the others revealed reasonable selectivity toward the tumorigenic cell.

\section{Pharmacophore modeling}

Elucidation of the binding approaches for the synthesized compounds is suggested based on finding the active structures. Table 1 shows the structure of the training set compounds (A1, A9, B1, B5, B9, C3, D5, E3, E5, F3, F5, G3, G5, H5, I3, and J5) as well as the test set compounds (B7, 
Table 1 Antiproliferative evaluation results of the synthesized combinatorial mixtures M1-M22 and schematic representation of an orthogonal deconvolution for prediction of the active compounds

\begin{tabular}{|c|c|c|c|c|c|c|c|c|c|c|c|c|c|}
\hline \multirow{2}{*}{$\begin{array}{l}\text { Hydrazides } \\
\text { Isatins }\end{array}$} & \multirow{2}{*}{ A } & \multirow[t]{2}{*}{ B } & \multirow[t]{2}{*}{ C } & \multirow[t]{2}{*}{ D } & \multirow[t]{2}{*}{$E$} & \multirow[t]{2}{*}{$F$} & \multirow[t]{2}{*}{ G } & \multirow[t]{2}{*}{$\mathrm{H}$} & \multirow[t]{2}{*}{ I } & \multirow[t]{2}{*}{$J$} & \multirow[t]{2}{*}{ K } & \multirow[b]{2}{*}{ Set 1} & \multirow[b]{2}{*}{$I C_{50}^{a}(\mu \mathrm{M})$} \\
\hline & & & & & & & & & & & & & \\
\hline 1 & A1 & B1 & $\mathrm{C} 1$ & D1 & E1 & F1 & G1 & $\mathrm{H} 1$ & 11 & J1 & K1 & M1 & $7.1 \pm 0.04$ \\
\hline 2 & A2 & B2 & $\mathrm{C} 2$ & D2 & E2 & F2 & G2 & $\mathrm{H} 2$ & 12 & $J 2$ & K2 & M2 & $9.76 \pm 0.027$ \\
\hline 3 & A3 & B3 & $\mathrm{C} 3$ & D3 & E3 & F3 & G3 & H3 & 13 & J3 & K3 & M3 & $7.16 \pm 0.069$ \\
\hline 4 & A4 & B4 & $\mathrm{C} 4$ & D4 & E4 & F4 & G4 & $\mathrm{H} 4$ & 14 & $J 4$ & K4 & M4 & $6.63 \pm 0.02$ \\
\hline 5 & A5 & B5 & C5 & D5 & E5 & F5 & G5 & H5 & 15 & J5 & K5 & M5 & $4.53 \pm 0.028$ \\
\hline 6 & A6 & B6 & $\mathrm{C} 6$ & D6 & E6 & F6 & G6 & $\mathrm{H} 6$ & 16 & J6 & K6 & M6 & $4.48 \pm 0.015$ \\
\hline 7 & A7 & B7 & $\mathrm{C} 7$ & D7 & E7 & F7 & G7 & $\mathrm{H} 7$ & 17 & J7 & K7 & M7 & $5.09 \pm 0.01$ \\
\hline 8 & A8 & B8 & C8 & D8 & E8 & F8 & G8 & $\mathrm{H} 8$ & 18 & J8 & K8 & M8 & b \\
\hline 9 & A9 & B9 & C9 & D9 & E9 & F9 & G9 & $\mathrm{H} 9$ & 19 & J9 & K9 & M9 & $9.29 \pm 0.019$ \\
\hline 10 & A10 & B10 & $\mathrm{C} 10$ & D10 & E10 & F10 & G10 & $\mathrm{H} 10$ & 110 & $\mathrm{~J} 10$ & K10 & M10 & $9.76 \pm 0.018$ \\
\hline 11 & A11 & B11 & C11 & D11 & E11 & F11 & G11 & $\mathrm{H} 11$ & 111 & J11 & K11 & M11 & $14.57 \pm 0.05$ \\
\hline Set 2 & M12 & M13 & M14 & M15 & M16 & M17 & M18 & M19 & M20 & M21 & M22 & & \\
\hline$I_{50}^{a}(\mu M)$ & $12.02 \pm 0.026$ & b & $22.6 \pm 0.038$ & $4.85 \pm 0.018$ & $10.1 \pm 0.05$ & b & $9.64 \pm 0.003$ & $21.06 \pm 0.02$ & b & b & $4.51 \pm 0.003$ & & \\
\hline
\end{tabular}

${ }^{\mathrm{a}} \mathrm{C}_{50}$ : concentration of the compound $(\mu \mathrm{M})$ producing $50 \%$ cell growth inhibition after $48 \mathrm{~h}$ of compound exposure, as determined by the WST-1 assay. Each experiment was run at least two times, and the results are presented as average values \pm standard deviation. ${ }^{b}$ Compounds or mixtures having $\mathrm{IC}_{50}$ value $>100 \mu \mathrm{M}$. 
Table 2 Cytotoxicity activity of the synthesized individual compounds 1-11(A-K)

\begin{tabular}{|c|c|c|c|c|c|c|c|c|c|c|c|}
\hline \multirow[b]{2}{*}{ Compound } & \multicolumn{11}{|c|}{ Compound//I $C_{50}(\mu \mathrm{M})$} \\
\hline & $\mathrm{A} 1$ & B1 & C1 & D1 & E1 & F1 & G1 & $\mathrm{H} 1$ & 11 & J1 & K1 \\
\hline K562 cell line & $20.04 \pm 0.07$ & $42.3 \pm 0.03$ & $13.47 \pm 0.038$ & $11.5 \pm 0.01$ & $6 \pm 0.007$ & $7.7 \pm 0.02$ & $5.66 \pm 0.11$ & $15.37 \pm 0.02$ & $11.59 \pm 0.034$ & $>100$ & $>100$ \\
\hline MCF-12A cell line & $69.23 \pm 0.123$ & $49.39 \pm 0.16$ & $>300$ & $>300$ & $>300$ & $64.31 \pm 0.026$ & $16.61 \pm 0.181$ & $37.12 \pm 0.27$ & $>300$ & $>300$ & $>300$ \\
\hline SI & 3.45 & 1.17 & $>22.27$ & $>26.1$ & $>50.00$ & 8.4 & 2.9 & 2.42 & $>25.88$ & NA & NA \\
\hline Compound & $\mathrm{A} 2$ & B2 & $\mathrm{C} 2$ & D2 & E2 & F2 & G2 & $\mathrm{H} 2$ & 12 & $J 2$ & K2 \\
\hline K562 cell line & $49.13 \pm 0.095$ & $20.19 \pm 0.09$ & $29.82 \pm 0.139$ & $8.3 \pm 0.003$ & $7.76 \pm 0.07$ & $12.9 \pm 0.002$ & $5.72 \pm 0.1$ & $8.05 \pm 0.035$ & $8.67 \pm 0.027$ & $23.32 \pm 0.32$ & $>100$ \\
\hline MCF-12A cell line & $32.55 \pm 0.073$ & $>300$ & $>300$ & $14.78 \pm 0.4$ & $>300$ & $31.39 \pm 0.096$ & $16.62 \pm 0.182$ & $11.67 \pm 0.003$ & $>300$ & $17.91 \pm 0.62$ & $>300$ \\
\hline SI & 0.66 & $>14.86$ & $>10.06$ & 1.8 & $>38.66$ & 2.4 & 2.9 & 1.45 & $>34.60$ & 0.77 & NA \\
\hline Compound & A3 & B3 & $\mathrm{C} 3$ & D3 & E3 & F3 & G3 & H3 & 13 & J3 & K3 \\
\hline K562 cell line & $32.37 \pm 0.074$ & $19.35 \pm 0.019$ & $14.86 \pm 0.055$ & $13.86 \pm 0.01$ & $7.13 \pm 0.009$ & $14 \pm 0.08$ & $9.88 \pm 0.41$ & $>100$ & $15.81 \pm 0.11$ & $19.75 \pm 0.08$ & $>100$ \\
\hline MCF-12A cell line & $27.06 \pm 0.272$ & $>300$ & $>300$ & $258.09 \pm 0.03$ & $>300$ & $8.71 \pm 0.17$ & $13.22 \pm 0.034$ & $97.36 \pm 0.062$ & $27.87 \pm 0.07$ & $>300$ & $83.8 \pm 0.039$ \\
\hline SI & 0.84 & $>15.50$ & $>20.19$ & 18.6 & $>42.08$ & 0.62 & 1.3 & NA & 1.76 & $>15.19$ & NA \\
\hline Compound & A4 & B4 & $\mathrm{C} 4$ & D4 & E4 & F4 & G4 & $\mathrm{H} 4$ & 14 & J4 & K4 \\
\hline K562 cell line & $38.88 \pm 0.138$ & $51.51 \pm 0.09$ & $30.96 \pm 0.2$ & $9.98 \pm 0.009$ & $7.5 \pm 0.03$ & $16.28 \pm 0.015$ & $8.43 \pm 0.163$ & $24.21 \pm 0.023$ & $11.57 \pm 0.051$ & $14.52 \pm 0.08$ & $>100$ \\
\hline MCF-12A cell line & $9.39 \pm 0.152$ & $>300$ & $>300$ & $>300$ & $>300$ & $50.85 \pm 0.048$ & $12.50 \pm 0.216$ & $>300$ & $>300$ & $12.74 \pm 0.32$ & $78.29 \pm 0.31$ \\
\hline SI & 0.24 & $>5.82$ & $>9.69$ & $>30$ & $>40.00$ & 3.1 & 1.5 & $>12.39$ & $>25.93$ & 0.88 & NA \\
\hline Compound & A5 & B5 & C5 & D5 & E5 & F5 & G5 & H5 & 15 & J5 & K5 \\
\hline K562 cell line & $>100$ & $7.3 \pm 0.01$ & $23.9 \pm 0.26$ & $8.67 \pm 0.007$ & $10.3 \pm 0.001$ & $10.4 \pm 0.044$ & $6.5 \pm 0.001$ & $5.88 \pm 0.004$ & $24.11 \pm 0.255$ & $6.99 \pm 0.005$ & $>100$ \\
\hline MCF-12A cell line & $39.74 \pm 0.073$ & $>300$ & $>300$ & $80.05 \pm 0.40$ & $>300$ & $39.09 \pm 0.24$ & $9.1 \pm 0.003$ & $7.35 \pm 0.094$ & $>300$ & $5.6 \pm 0.003$ & $>300$ \\
\hline SI & NA & $>41.10$ & $>12.55$ & 9.2 & $>29.13$ & 3.8 & 1.4 & 1.25 & $>12.44$ & 0.80 & NA \\
\hline Compound & A6 & B6 & $\mathrm{C} 6$ & D6 & E6 & F6 & G6 & $\mathrm{H} 6$ & 16 & J6 & K6 \\
\hline K562 cell line & $>100$ & $>100$ & $>100$ & $8.87 \pm 0.017$ & $9.58 \pm 0.03$ & $13.5 \pm 0.03$ & $6.62 \pm 0.22$ & $6.49 \pm 0.017$ & $8.55 \pm 0.06$ & $7.19 \pm 0.051$ & $>100$ \\
\hline MCF-12A cell line & $13.91 \pm 0.003$ & $>300$ & $>300$ & $134.18 \pm 0.142$ & $>300$ & $9.6 \pm 0.15$ & $13.05 \pm 0.20$ & $34.77 \pm 0.062$ & $26.15 \pm 0.14$ & $10.16 \pm 1.2$ & $>300$ \\
\hline SI & NA & NA & NA & 15.1 & $>31.32$ & 0.71 & 2 & 5.36 & 3.06 & 1.41 & NA \\
\hline Compound & A7 & B7 & C7 & D7 & E7 & F7 & G7 & $\mathrm{H7}$ & 17 & $J 7$ & K7 \\
\hline K562 cell line & $>100$ & $47.25 \pm 0.027$ & $>100$ & $9.1 \pm 0.026$ & $8.9 \pm 0.02$ & $12.86 \pm 0.01$ & $7.68 \pm 0.34$ & $23.67 \pm 0.39$ & $>100$ & $18.02 \pm 0.13$ & $>100$ \\
\hline MCF-12A cell line & $11.03 \pm 0.151$ & $>300$ & $>300$ & $>300$ & $>300$ & $11.39 \pm 0.25$ & $12.98 \pm 0.439$ & $73.18 \pm 0.072$ & $>300$ & $>300$ & $61.34 \pm 0.01$ \\
\hline SI & NA & $>6.35$ & NA & $>33$ & $>33.71$ & 0.89 & 1.7 & 3.09 & NA & $>16.65$ & NA \\
\hline Compound & A8 & B8 & $\mathrm{C} 8$ & D8 & E8 & F8 & G8 & $\mathrm{H} 8$ & 18 & J8 & K8 \\
\hline K562 cell line & $>100$ & $27.23 \pm 0.092$ & $>100$ & $6.9 \pm 0.001$ & $10.34 \pm 0.015$ & $6.99 \pm 0.007$ & $15.12 \pm 0.48$ & $40.65 \pm 0.24$ & $>100$ & $45.51 \pm 0.46$ & $>100$ \\
\hline MCF-12A cell line & $10.54 \pm 0.157$ & $>300$ & $>300$ & $>300$ & $>300$ & $21.29 \pm 0.104$ & $>300$ & $>300$ & $>300$ & $>300$ & $>300$ \\
\hline SI & NA & $>11.02$ & NA & $>43.5$ & $>29.01$ & 3 & $>19.8$ & $>7.38$ & NA & $>6.59$ & NA \\
\hline Compound & A9 & B9 & C9 & D9 & E9 & F9 & G9 & $\mathrm{H} 9$ & 19 & J9 & K9 \\
\hline
\end{tabular}


Table 2 Cytotoxicity activity of the synthesized individual compounds 1-11(A-K) (Continued)

\begin{tabular}{|c|c|c|c|c|c|c|c|c|c|c|c|}
\hline K562 cell line & $90.67 \pm 0.19$ & $17.42 \pm 0.05$ & $>100$ & $6.2 \pm 0.01$ & $9.77 \pm 0.01$ & $6.77 \pm 0.01$ & $>100$ & $26.72 \pm 0.38$ & $31.19 \pm 0.004$ & $52.05 \pm 0.15$ & $>100$ \\
\hline MCF-12A cell line & $14.48 \pm 0.647$ & $>300$ & $>300$ & $>300$ & $>300$ & $13.14 \pm 0.114$ & $>300$ & $>300$ & $>300$ & $>300$ & $5.88 \pm 0.11$ \\
\hline SI & 0.16 & $>17.22$ & NA & $>48.4$ & $>30.71$ & 1.9 & NA & $>11.23$ & $>9.62$ & $>5.76$ & NA \\
\hline Compound & A10 & B10 & $\mathrm{C} 10$ & D10 & E10 & F10 & G10 & $\mathrm{H} 10$ & 110 & J10 & K10 \\
\hline K562 cell line & $>100$ & $17.88 \pm 0.062$ & $>100$ & $8.9 \pm 0.43$ & $8.66 \pm 0.06$ & $6.3 \pm 0.005$ & $8.43 \pm 0.18$ & $23.34 \pm 0.19$ & $19.14 \pm 0.07$ & $>100$ & $88.85 \pm 0.26$ \\
\hline MCF-12A cell line & $8.33 \pm 0.044$ & $>300$ & $>300$ & $12.57 \pm 0.089$ & $>300$ & $11.01 \pm 0.11$ & $14.19 \pm 0.175$ & $32.86 \pm 0.065$ & $>300$ & $>300$ & $51.02 \pm 0.04$ \\
\hline SI & NA & $>16.78$ & NA & 1.4 & $>34.64$ & 1.7 & 1.7 & 1.41 & $>15.67$ & NA & 0.57 \\
\hline Compound & A11 & B11 & C11 & D11 & E11 & F11 & G11 & $\mathrm{H} 11$ & 111 & J11 & K11 \\
\hline K562 cell line & $>100$ & $39.24 \pm 0.006$ & $>100$ & $9.11 \pm 0.23$ & $13.65 \pm 0.52$ & $5.92 \pm 0.002$ & $>100$ & $8.46 \pm 0.27$ & $>100$ & $>100$ & $>100$ \\
\hline MCF-12A cell line & $7.88 \pm 0.01$ & $>300$ & $>300$ & $10.15 \pm 0.072$ & $>300$ & $12.46 \pm 0.053$ & $97.93 \pm 0.16$ & $29.84 \pm 0.17$ & $>300$ & $>300$ & $28.31 \pm 0.14$ \\
\hline SI & NA & $>7.65$ & NA & 1.1 & $>21.98$ & 2.1 & NA & 3.53 & NA & NA & NA \\
\hline
\end{tabular}

${ }^{\mathrm{a}} \mathrm{C}_{50}$ : concentration of the compound $(\mu \mathrm{M})$ producing $50 \%$ cell growth inhibition after $48 \mathrm{~h}$ of compound exposure, as determined by the WST-1 assay. Each experiment was run at least two times, and the results are presented as average values \pm standard deviation. 
B8, B10, D2-D4, E2, F2, H8, J4, and J8). Based on the assumption that the active compounds bind in a similar fashion at the active site. Ligandscout program [33] was employed to evaluate the common features essential for antiproliferative activity and the hypothetical geometries adopted by these ligands in their most active forms. Thus, these compounds were submitted for pharmacophore model generation based on the shared chemical features. Diverse conformations within $20 \mathrm{kcal} / \mathrm{mol}$ energy range were generated and submitted to the alignment procedure.

The successful pharmacophore run resulted in generation of 10 hypotheses (Hypo1-10, Table 3). Hypo1-7 composed of two hydrophobes, three hydrogen bond acceptors and two hydrogen bond donors. According to its highest rank score and mapping into all training set molecules, hypo1 was considered statistically as the best hypothesis and was selected for further investigation and analysis. The top-ranked chemical feature-based pharmacophore model identified in this study is shown in Figure 1. This pharmacophore model contains seven chemical features: two hydrophobes (orange), three hydrogen bond acceptors (red) and two hydrogen bond donors (green).

All the training set and test set compounds were mapped onto hypo1 with scoring the orientation of a mapped compound within the hypothesis features using a "fit value" score. As a quick and primary validation of hypo1, mapping of the compounds found to show a good agreement between the fit value and the biological activity (Tables 4 and 5, Figures 2 and 3).

Initial investigation of the results shown in Tables 4 and 5 revealed a moderate correlation between the fit value and the biological activity of each of the tested compounds. The highly active compounds showed a range of fit value of 81.54-80.03 where the moderately active derivatives showed a lower fit value average of 74.0. This initial correlation encouraged us to generate a

Table 3 Summary of the generated pharmacophores of the Antiproliferative activities of the synthesized Schiff bases against MCF-12A cell line

\begin{tabular}{lll}
\hline Hypothesis & Features $^{*}$ & Rank score \\
\hline Hypo1 & HHAAADD & 0.8665 \\
Hypo2 & HHAAADD & 0.8657 \\
Hypo3 & HHAAADD & 0.8656 \\
Hypo4 & HHAAADD & 0.8652 \\
Hypo5 & HHAAADD & 0.8651 \\
Hypo6 & HHAAADD & 0.8624 \\
Hypo7 & HHAAADD & 0.8622 \\
Hypo8 & HHRAAADD & 0.8578 \\
Hypo9 & HHRAAADD & 0.8550 \\
Hypo10 & HHRAAADD & 0.8483 \\
\hline
\end{tabular}

${ }^{*} H$ hydrophobic, $R$ aromatic ring, $A$ hydrogen bond acceptor, $D$ hydrogen bond donor.

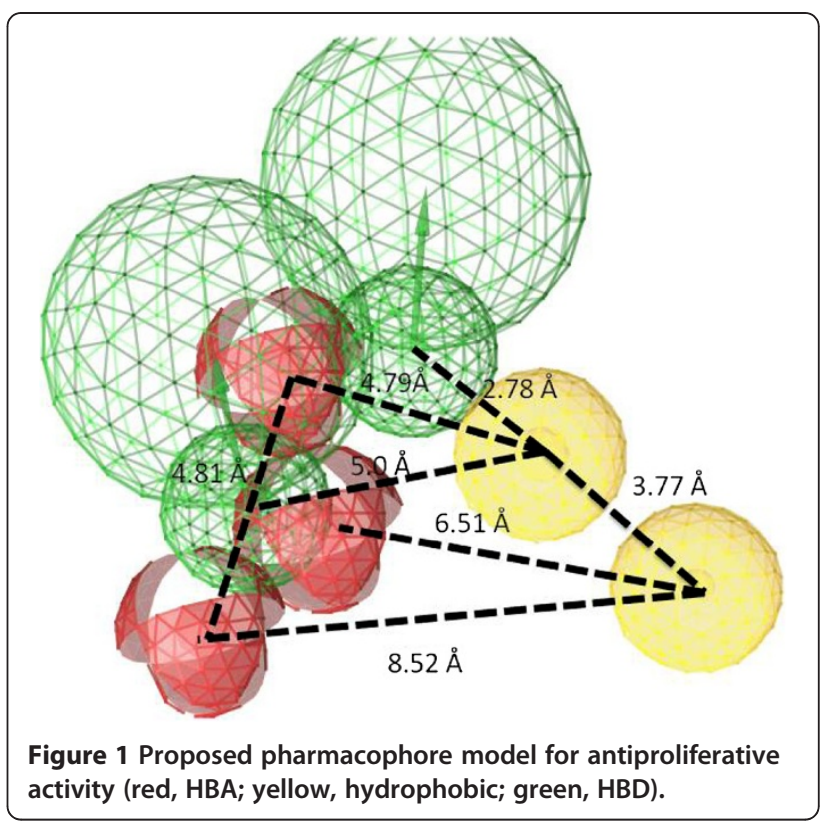

linear model based on "fit value" to predict the biological activity of the compounds under investigation. The generated model (Equation 1) showed good statistics and was used successfully to calculate the activity of the tested compounds (Table 4).

$$
\mathrm{pIC}_{50}=7.4164 \text { fit value }+65.291
$$

$\mathrm{n}=16$, st. error $=0.181, \mathrm{R}=0.834, \mathrm{R}^{2}=0.696$ Where $\mathrm{n}$ : number of compounds; R: multiple correlation coefficient

Table 4 Output for Hypo1 mapping and predictive model of training set compounds

\begin{tabular}{llllll}
\hline Compds & $I_{\mathbf{5 0}}(\boldsymbol{\mu M})$ & $\mathbf{p}\left(\mathrm{IC}_{\mathbf{5 0}} \times \mathbf{1 0}^{\mathbf{3}}\right)$ & Fit value & $\begin{array}{l}\text { Predicted } \\
\mathbf{p}\left(\mathbf{I C}_{\mathbf{5 0}} \times \mathbf{1 0}^{-\mathbf{3}}\right)\end{array}$ & Residuals \\
\hline A1 & 20.04 & 1.70 & 74.84 & 1.47 & 0.23 \\
A9 & 90.67 & 1.04 & 74.46 & 1.44 & -0.40 \\
B1 & 42.30 & 1.37 & 74.84 & 1.47 & -0.10 \\
B5 & 7.30 & 2.14 & 81.42 & 2.09 & 0.05 \\
B9 & 17.42 & 1.76 & 74.46 & 1.44 & 0.32 \\
C3 & 14.86 & 1.83 & 80.39 & 1.99 & -0.16 \\
D5 & 8.67 & 2.06 & 81.54 & 2.10 & -0.04 \\
E3 & 7.13 & 2.15 & 80.37 & 1.99 & 0.16 \\
E5 & 10.30 & 1.99 & 80.42 & 2.00 & -0.01 \\
F3 & 14.00 & 1.85 & 80.37 & 1.99 & -0.14 \\
F5 & 10.40 & 1.98 & 80.42 & 2.00 & -0.02 \\
G3 & 9.88 & 2.01 & 80.44 & 2.00 & 0.01 \\
G5 & 6.50 & 2.19 & 81.52 & 2.10 & 0.09 \\
H5 & 5.88 & 2.23 & 81.54 & 2.10 & 0.13 \\
I3 & 15.81 & 1.80 & 80.44 & 2.00 & -0.20 \\
J5 & 6.99 & 2.16 & 81.54 & 2.10 & 0.06 \\
\hline & & & & & \\
\hline
\end{tabular}




\begin{tabular}{|c|c|c|c|c|c|}
\hline Compds & $\mathrm{IC}_{50}(\mu \mathrm{M})$ & $\mathrm{p}\left(\mathrm{IC} \mathrm{C}_{50} \times 10^{-3}\right)$ & Fit value & $\begin{array}{l}\text { Predicted } \\
\mathrm{p}\left(\mathrm{IC}_{50} \times 10^{-3}\right)\end{array}$ & Residuals \\
\hline B7 & 47.25 & 1.33 & 68.76 & 1.30 & 0.03 \\
\hline B8 & 27.23 & 1.56 & 68.78 & 1.30 & 0.26 \\
\hline B10 & 17.88 & 1.75 & 74.2 & 1.58 & 0.17 \\
\hline D2 & 8.30 & 2.08 & 80.71 & 1.92 & 0.16 \\
\hline D3 & 13.86 & 1.86 & 80.39 & 1.90 & -0.04 \\
\hline D4 & 9.98 & 2.00 & 80.56 & 1.91 & 0.09 \\
\hline E2 & 7.76 & 2.11 & 80.71 & 1.92 & 0.19 \\
\hline F2 & 12.90 & 1.89 & 80.71 & 1.92 & -0.03 \\
\hline H8 & 40.65 & 1.39 & 68.78 & 1.30 & 0.09 \\
\hline J4 & 14.52 & 1.84 & 80.56 & 1.91 & -0.07 \\
\hline J8 & 45.51 & 1.34 & 68.78 & 1.30 & 0.04 \\
\hline
\end{tabular}

Figures $4 \mathrm{a}-\mathrm{c}$ showed the alignment of the hypothesis model with compounds $\mathbf{H 5}, \mathbf{A 6}$, and $\mathbf{I 8}$ as representative examples. A closer look at the mapped structures revealed the importance of certain structural features for activity. The substituted benzene ring of the isatin scaffold is thought to be critical for activity where the slight displacement of its fused benzene ring away from hydrophobic pharmacophore center (Figure 4b) or displacement of the isatin nitrogen away from the hydrogen bond donor pharmacophore center (Figure 4c) can partially explain their lack of activity. Furthermore, lack of antiproliferative activity of the derivatives containing 5-unsubstituted isatin moiety A1-K1 could be attributed to their missing of one of the essential hydrophobic pharmacophore centers (Figure 4c). The rest of the features that are common for all compounds are the oxygen atom at 2-position of the isatin, the hydrazone nitrogen as hydrogen bond acceptor, and the hydrazide $\mathrm{NH}$ as hydrogen bond donor.

\section{Docking procedure}

Docking study was undertaken using Dock6.4 [34] in order to investigate the possible interactions between the designed

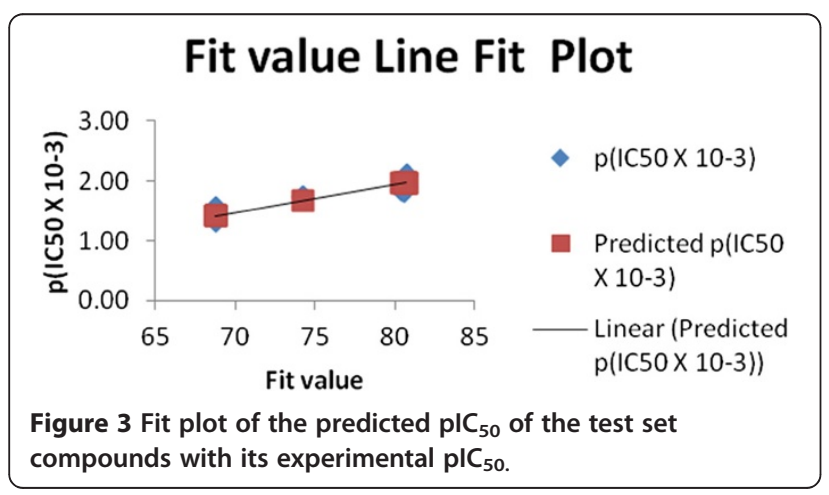

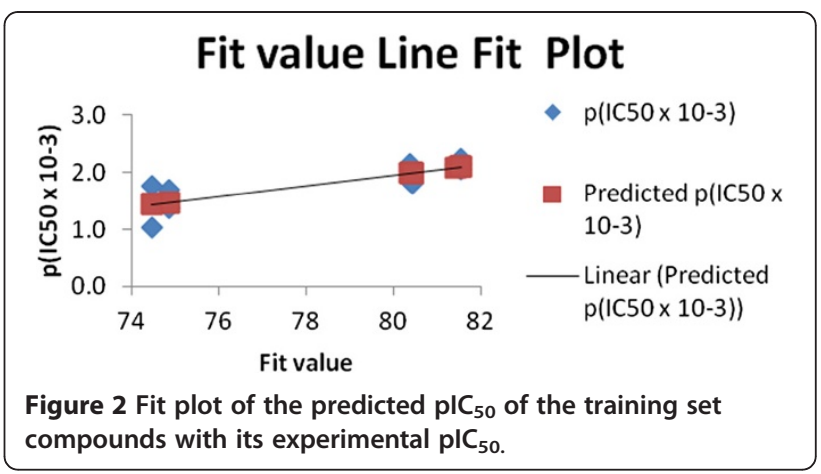

compounds and the active site of the epidermal growth factor receptor (EGFR) kinase and to compare it with the binding mode of the known EGFR inhibitor $N$-[4-(3bromophenylamino)quinazolin-6-yl]acrylamide

(DJK_3021_ A). The X-ray structure of the enzyme bounded with DJK_3021_A was taken from the protein data bank; PDB code: 2J5F [35]. The RMSD value difference of $1.005 \AA$ of the pose of the non-restricted redocking of the X-ray structure of the EGFR inhibitor (DJK_3021_A) from itself also confirmed the approach (Figure 5). The binding site includes hydrophobic pocket delineated by the side chains of Leu16, Phe21, Val24, Ala36, Lys38, Glu51, Leu76, Leu80, Cys85, Leu116, Asp127 (Figure 5).

The docking poses of compound $\mathbf{H 5}$, as an example of the designed compounds (Figures 6 and 7), showed that isatin scaffold structure is oriented in the binding site as the same as the quinazoline moiety of the DJK_3021_A Xray structure with displacement of the hydrogen bond acceptor atom in compound $\mathbf{H} \mathbf{5}$ from those of the quinazoline ring of the DJK_3021_A. The isatin scaffold is oriented in the hydrophobic pocket surrounded by the side chains of Leu16, Phe21, Leu80 and Leu116. The hydrophobic portion of trifluoromethoxy group is overlaid with the bromophenyl moiety of the DJK_3021_A whereas it is stabilized between Val24 and Lys38 with the hydrophobic interactions. Moreover, hydrophobic aromatic substituent at nitrogen atom of isatin in $\mathbf{H 5}$ is aligned with the hydrophobic portion of acrylamide substituent of the DJK_3021_A whereas it is positioned in parallel orientation between its pi system and Leu116 with additional hydrophobic interaction between the aromatic ring, of $\mathrm{N}$ benzoyl moiety, and Phe21 side chain.

Comparing the docking poses of the designed derivatives with DJK_3021_A, it could be postulated that the designed compounds might act on the same enzyme target where DJK_3021_A acted.

\section{Conclusions}

A combinatorial library of 121 Schiff bases of indoline2,3-dione (isatin) was investigated for their potential 
(a)

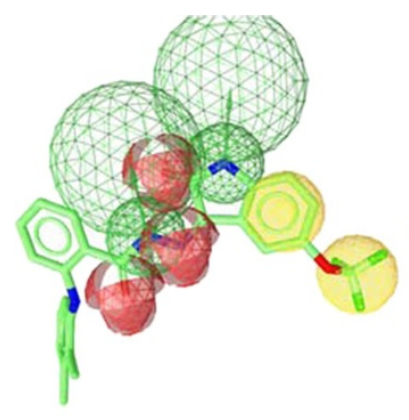

(b)

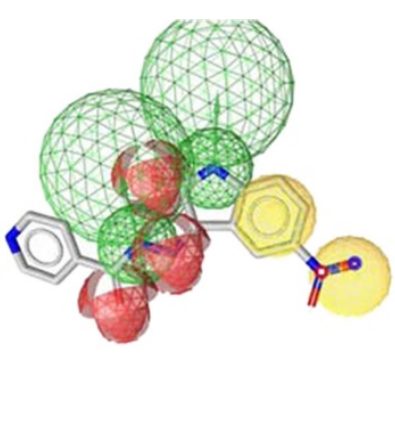

(c)

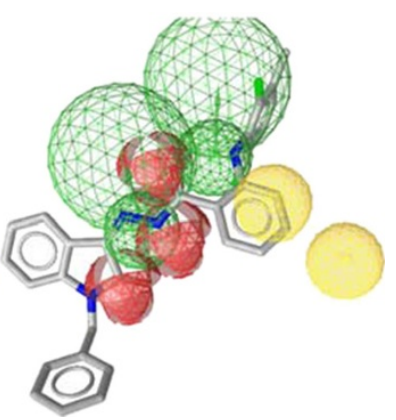

Figure 4 (a) Best aligned pose of compound $\mathrm{H} 5\left(\mathrm{IC}_{50}=\mathbf{5 . 8 8} \mu \mathrm{M}\right)$ superposed with the query (Hypo1). (b) Best aligned pose of compound A6 $\left(I C_{50}>100 \mu \mathrm{M}\right)$ fitted inadequately with the query (Hypo1). (c) Best aligned pose of weak $\mathbf{I} 8\left(\mathrm{IC}_{50}>100 \mu \mathrm{M}\right)$ overlaid onto the pharmacophore model (Hypo1).

antiproliferative activity. Potent activity was observed with some of these derivatives against K562 chronic myelogenous leukemia cells with considerable selectivity compared with the non-tumorigenic MCF-12A cell line. Pharmacophore modeling study revealed that these compounds are able to effectively satisfy the proposed common feature sites using energy accessible conformers $\left(\mathrm{E}_{\mathrm{conf}}<20 \mathrm{kcal} / \mathrm{mol}\right)$. Also, docking study could suggest the similarity in binding mode of the designed compounds and DJK_3021_A with the EGFR kinase in its X-ray structure.

\section{Experimental}

Isatins and hydrazides building blocks were obtained either commercially or synthesized along with the designed target Schiff bases according to the reported literatures [26,27,29-31]. Cytotoxicity was done at Stem Cell Therapy Program, King Faisal Specialized Hospital and Research Center, Riyadh-Saudi Arabia.

\section{Cell cytotoxicity assay}

K562 chronic myelogenous leukemia cells were purchased from the American Type Culture Collection. Cells were maintained in RPMI 1640 (Sigma), supplemented with 10\% FCS (Cambrex Bio Science), 100 IU/ $\mathrm{mL}$ penicillin, $100 \mathrm{mg} / \mathrm{mL}$ streptomycin and $2 \mathrm{mmol} /$ L L-glutamine (Sigma) and were used to investigate the cytotoxicity of all the synthesized compounds.

MCF-12A cell line is a non-tumorigenic epithelial cell line established from tissue taken at reduction

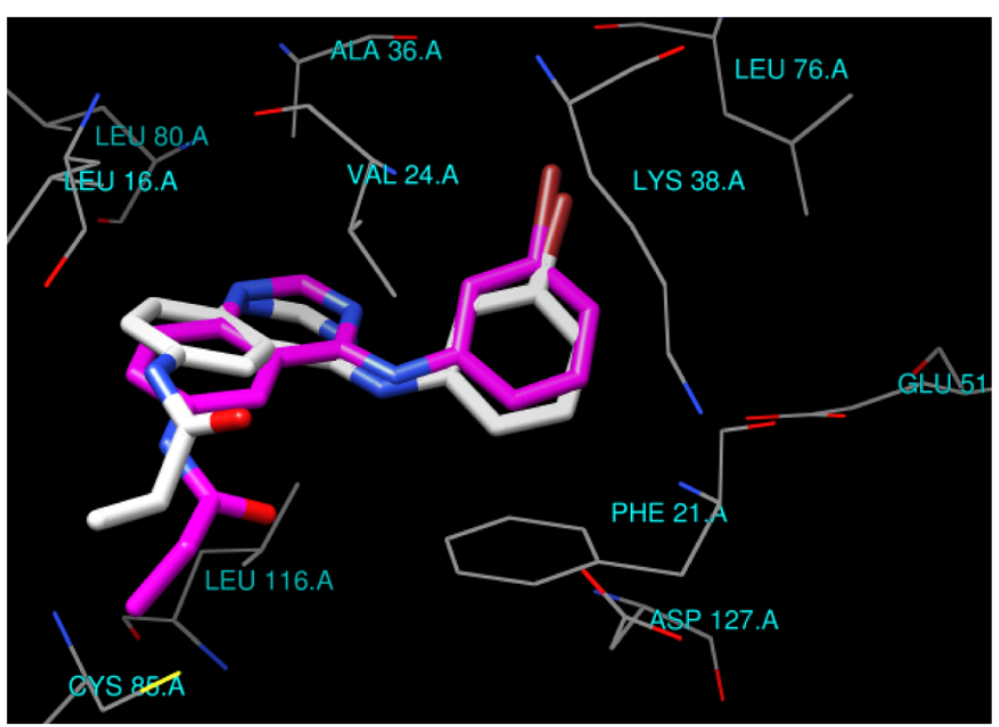

Figure $5 \mathrm{~N}$-[4-(3-bromophenylamino)quinazolin-6-yl]acrylamide (DJK_3021_A) from 2J5F (X-ray magenta, docked white) oriented epidermal growth factor receptor (EGFR) kinase binding site. 


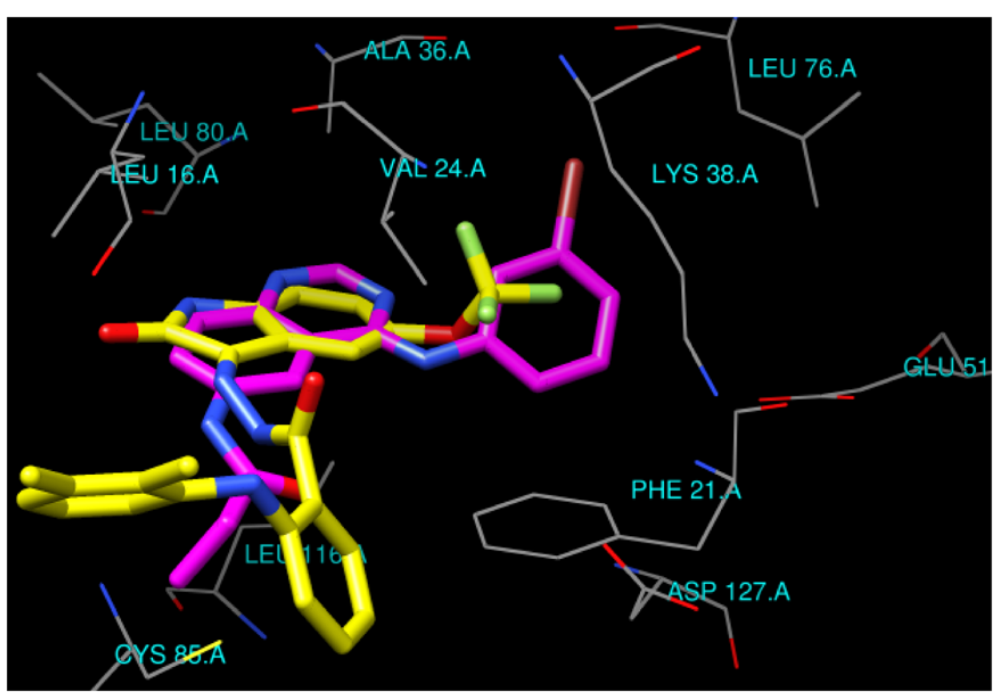

Figure 6 Compound H5 (colored Yellow) docked in EGFR binding site and overlaid with DJK_3021_A x-ray structure (colored magenta).

mammoplasty from a nulliparous patient with fibrocystic breast disease that contained focal areas of intraductal hyperplasia and were used to investigate the cytotoxicity of all the synthesized compounds.

Cells were seeded into 96 -well plates at $0.4 * 10^{4} /$ well and incubated overnight. The medium was replaced with fresh one containing the desired concentrations of the compounds. After $48 \mathrm{~h}, 10 \mu \mathrm{l}$ of the WST-1 reagent were added to each well and the plates were reincubated for $4 \mathrm{~h}$ at $37^{\circ} \mathrm{C}$. The amount of formazan was quantified using ELISA reader at $450 \mathrm{~nm}$.

\section{Selectivity index (SI)}

In the present study, the degree of selectivity of the synthetic compounds is expressed as per the previous

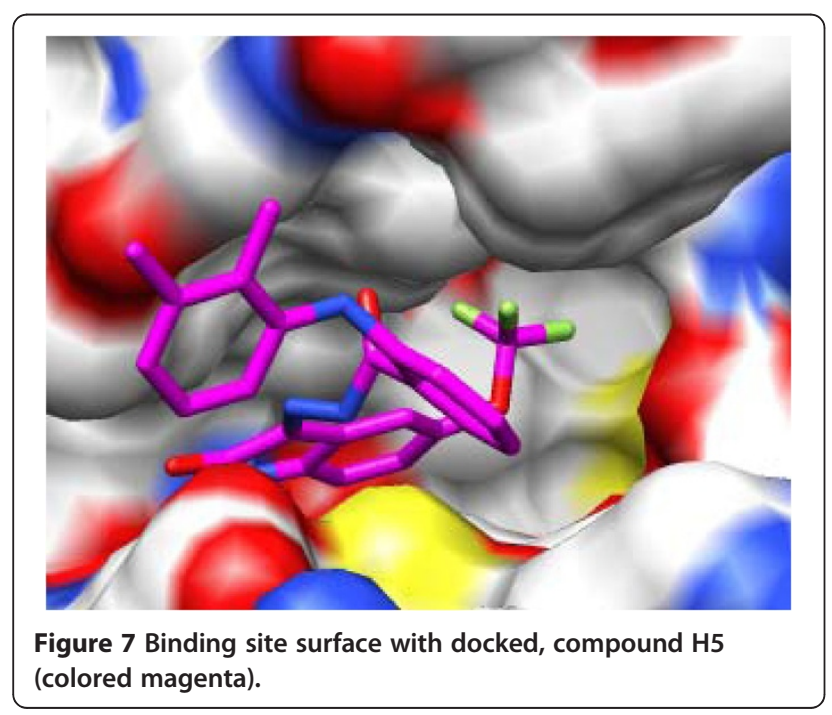

reports [36,37]: $\mathrm{SI}=\mathrm{IC}_{50}$ of pure compound in a normal cell line/ $\mathrm{IC}_{50}$ of the same pure compound in cancer cell line, where $\mathrm{IC}_{50}$ is the concentration required to kill $50 \%$ of the cell population.

\section{Molecular modeling}

\section{Ligand based pharmacophore modeling}

The study was carried out using the software LigandScout (version 3.0). LigandScout program was used to derive the $3 \mathrm{D}$ chemical feature-based pharmacophores from the structural data of the synthesized compounds (Table 1) using default settings [33]. Compounds A1, A6-A9, B1, B5-B10, C3, C6-C8, D2-D5, E2, E3, E5, F2, F3, F5, G3, G5, G9, H5, H8, $\mathrm{I3}, \mathrm{I8}, \mathrm{J4}, \mathrm{J5}, \mathrm{J8}$, and $\mathrm{K} 6$ are included in the modeling method. Prior to the generation of pharmacophore hypotheses, the training set compounds (A1, A9, B1, B5, B9, C3, D5, E3, E5, F3, F5, G3, G5, H5, I3, and J5) were converted to 3D structure and were used to generate diverse conformations. Diverse Conformation Generation protocol implemented in LigandScout program was used to generate conformations using the best conformation model generation method. Other parameters like maximum number of 500 conformers, and an energy threshold value of $20 \mathrm{kcal} / \mathrm{mol}$ above the global energy minimum were chosen during conformation generation. During pharmacophore hypothesis generation four pharmacophoric features like hydrogen bond acceptor (HBA), hydrogen bond donor (HBD), ring aromatic (RA) and hydrophobic (HY) were selected based on the feature mapping results. All parameters were set to their default values. 


\section{Pharmacophore validation}

The generated pharmacophore hypothesis was validated using leave-one-out and test set methods.

\section{Leave-one-out method}

The pharmacophore hypothesis is cross validated by leave-one-out method. In this method, one compound is left in the generation of a new pharmacophore model and its affinity is predicted using that new model. The model building and estimation cycle were repeated until each compound was left out once [38]. This test was performed to verify whether the correlation coefficient of the training set compounds is strongly depend on one particular compound or not [39].

\section{Test set method}

Compounds B7, B8, B10, D2-D4, E2, F2, H8, J4, and $\mathbf{J 8}$ were selected as test set compounds. This method is used to elucidate whether the generated pharmacophore hypothesis is proficient to predict the activities of the compounds other than training set and classify them correctly in their activity scale. The conformation generation for test set compounds was carried out in a similar way like training set compounds using conformation analysis algorithm. The compounds associated with their conformations were subsequently carried out for pharmacophore mapping using Ligand Pharmacophore Mapping protocol with Best/Flexible Search option.

\section{Docking procedure}

All molecular modeling studies were performed on PC windows Vista Home Premium Intel(R) Core(TM) 2 Duo, $1.83 \mathrm{GHz}$ using Dock 6.4 [34]. All compounds were generated in the protonation state under physiological condition. The coordinates of the X-ray structure of the epidermal growth factor receptor (EGFR) kinase domain in complex with an irreversible inhibitor DJK_3021_A (PDB code: 2J5F) was taken from the Protein Data Bank [35]. The co-crystallized ligand was docked in its original protein structure. Docking was performed with default settings to obtain a population of possible conformations and orientations for the ligands at the binding site. A $10 \AA$ sphere around the centre of the binding pocket was defined as binding pocket for the docking runs. All torsion angles in each compound were allowed to rotate freely.

\section{Additional file}

Additional file 1: Antiproliferative evaluation results of the synthesized combinatorial mixtures $\mathbf{M} 1-\mathbf{M} 2 \mathbf{2}$ and schematic representation of an orthogonal deconvolution for prediction of the active compounds.
Competing interests

The authors declare that they have no competing interests.

\section{Authors' contributions}

TA has formulated the research idea, result's interpretation and discussion and prepared the manuscript, AAR undertook the molecular modeling studies, result's interpretation and shared in preparation of the manuscript, MIA participated in result's interpretation and shared in preparation of the manuscript, AA carried out antiproliferative investigations, HAA participated in preparation of the manuscript. All authors have read and approved the final manuscript.

\section{Acknowledgements}

This research project is supported by "NPST program by King Saud University", Project Number MED598-02-08.

\section{Author details}

'Department of Pharmaceutical Chemistry, College of Pharmacy, King Saud University, P.O. Box 2457, Riyadh 11451, Saudi Arabia. ${ }^{2}$ Faculty of Pharmacy, Assiut University, Assiut 71526, Egypt. 3Kayyali Chair, Pharmaceutical Technology center, College of Pharmacy, King Saud University, P.O. Box 2457, Riyadh 11451, Saudi Arabia. ${ }^{4}$ Stem Cell Therapy Program, King Faisal Specialized Hospital and Research Center, P.O. Box 3354, Riyadh 11211, Saudi Arabia.

Received: 11 March 2012 Accepted: 8 May 2012

Published: 30 May 2012

\section{References}

1. Ahmedin J, Siegel R, Ward E, Hao Y, Xu J, Murray T, Thun MJ: Cancer Statistics, 2008. CA: A Cancer J Clin 2008, 58:71-96.

2. Sinha R, El-Bayoumy K: Apoptosis is a critical cellular event in cancer chemoprevention and chemotherapy by selenium compounds. Current Cancer Drug Targets 2004, 4:13-28.

3. Cozzi P, Mongelli N, Suarato A: Recent anticancer cytotoxic agents. Curr Med Chem Anti-Cancer Agents 2004, 4:93-121.

4. Rew DA, Wilson GD: Cell production rates in human tissues and tumours and their significance. Part II: clinical data. Eur J Surg Oncol 2000, 26:405-417

5. Chabner BA, Roberts TG Jr: Chemotherapy and the war on cancer. Nat Rev Cancer 2005, 5:65-72.

6. Kamb A, Wee $\mathrm{S}$, Lengauer $\mathrm{C}$ : Why is cancer drug discovery so difficult? Nat Rev Drug Disc 2007, 6:115-120.

7. Onnis V, Cocco MT, Fadda R, Congiu C: Synthesis and evaluation of anticancer activity of 2-arylamino-6-trifluoromethyl-3(hydrazonocarbonyl)pyridines. Bioorg Med Chem 2009, 17:6158-6165.

8. Kamal A, Naseer M, Khan A, Reddy KS, Rohini K. Synthesis of a new class of 2-anilino substituted nicotinylarylsulfonylhydrazides as potential anticancer and antibacterial agents. Bioorg Med Chem 2007, 15:1004-1013.

9. Easmon J, Purstinger G, Thies KS, Heinisch G, Hofmann J: Synthesis, structure-activity relationships, and antitumor studies of 2-benzoxazolyl hydrazones derived from alpha- $(N)$-acyl heteroaromatics. J Med Chem 2006, 49:6343-6350.

10. Xia Y, Fan CD, Zhao BX, Zhao J, Shin DS, Miao JY: Synthesis and structure-activity relationships of novel 1-arylmethyl-3-aryl- $1 \mathrm{H}$ pyrazole-5-carbohydrazide hydrazone derivatives as potential agents against A549 lung cancer cells. Eur J Med Chem 2008, 43:2347-2353.

11. Vogel S, Kaufmann D, Pojarová M, Müller C, Pfaller T, Kühne S, Bednarski PJ, Angerer Ev: Aroyl hydrazones of 2-phenylindole-3-carbaldehydes as novel antimitotic agents. Bioorg Med Chem 2008, 16:6436-6447.

12. Putt KS, Chen GW, Pearson JM, Sandhorst JS, Hoagland MS, Kwon JT, Hwang SK, Jin H, Churchwell MI, Cho MH, Doerge DR, Helferich WG, Hergenrother PJ: Small-molecule activation of procaspase-3 to caspase-3 as a personalized anticancer strategy. Nat Chem Biol 2006 10:543-550.

13. Xu G, Abad MC, Connolly PJ, Neeper MP, Struble GT, Springer BA, Emanuel SL, Pandey N, Gruninger RH, Adams M, Moreno-Mazza S, Fuentes-Pesquera AR, Middleton SA: 4-Amino-6-arylamino-pyrimidine-5-carbaldehyde hydrazones as potent ErbB-2/EGFR dual kinase inhibitors. Bioorg Med Chem Lett 2008, 18:4615-4619.

14. Horiuchi T, Chiba J, Uoto K, Soga T: Discovery of novel thieno[2,3- $d$ ] pyrimidin-4-yl hydrazone-based inhibitors of Cyclin D1-CDK4: Synthesis, 
biological evaluation, and structure-activity relationships. Bioorg Med Chem Lett 2009, 19:305-308.

15. Hellmuth K, Grosskopf S, Lum CT, Wurtele M, Roder N, Kries JPv, Rosario M, Rademann R, Birchmeier J: Specific inhibitors of the protein tyrosine phosphatase Shp2 identified by high-throughput docking. PNAS 2008, 105:7275-7280.

16. Hofmann J, Easmon J, Puerstinger G, Heinisch G, Jenny M, Shtil AA, Hermann M, Condorelli DF, Sciré S, Musumarra G: N-benzoxazol-2-yl- $N$ '-1-(isoquinolin-3-yl-ethylidene)-hydrazine, a novel compound with antitumor activity, induces radicals and dissipation of mitochondrial membrane potential. Invest New Drugs 2009, 27:189-202.

17. Popp FD, Pajouhesh H: Potential anticonvulsants VI: Condensation of isatins with cyclohexanone and other cyclic ketones. J Pharm Sci 1983, 72:318-321.

18. Vine KL, Locke JM, Ranson M, Benkendorff K, Pyne SG, Bremner JB: In vitro cytotoxicity evaluation of some substituted isatin derivatives. Bioorg Med Chem 2007, 15:931-938.

19. Vine $K L$, Locke JM, Ranson M, Pyne SG, Bremner JB: An investigation into the cytotoxicity and mode of action of some novel $\mathrm{N}$-alkyl-substituted isatins. J Med Chem 2007, 50:5109-5117.

20. Matesic L, Locke JM, Bremner JB, Pyne SG, Skropeta D, Ranson M, Vine KL: $\mathrm{N}$-Phenethyl and $\mathrm{N}$-naphthylmethyl isatins and analogues as in vitro cytotoxic agents. Bioorg Med Chem 2008, 16:3118-3124.

21. Prenen H, Cools J, Mentens N, Folens C, Sciot R, Schoffski P, Van Oosterom A, Marynen P, Debiec-Rychter M: Efficacy of the kinase inhibitor SU11248 against gastrointestinal stromal tumor mutants refractory to imatinib mesylate. Clin Cancer Res. 2006, 8:2622-2627.

22. Motzer RJ, Michaelson MD, Redman BG, Hudes GR, Wilding G, Figlin RA, Ginsberg MS, Kim ST, Baum CM, DePrimo SE, Li JZ, Bello CL, Theuer CP, George DJ, Rini BI: Activity of SU11248, a multitargeted inhibitor of vascular endothelial growth factor receptor and plateletderived growth factor receptor, in patients with metastatic renal cell carcinoma. J Clin Oncol 2006, 24:16-24.

23. Hössel R, Leclerc $S$, Endicott JA, Nobel M, Lawrie A, Tunnah $P$, Leost ME, Damiens E, Marie D, Marko D, Niederberger E, Tang WC, Eisenbrand G, Meijer L: Indirubin, the active constituent of a Chinese antileukaemia medicine, inhibits cyclin-dependent kinases. Nat Cell Biol 1999, 1:60-67.

24. Nam S, Buettner R, Turkon J, Kim D, Cheng JQ, Muehlbeyer S, Hippe F, Vatter S, Merz K-H, Eisenbrand G, Jove R: Indirubin derivatives inhibit Stat3 signaling and induce apoptosis in human cancer cells. Proc Natl Acad Sci USA 2005, 102:5998-6003.

25. Chu W, Rothfuss J, Zhou D, Mach RH: Synthesis and evaluation of isatin analogs as caspase- 3 inhibitors: introduction of a hydrophilic group increases potency in a whole cell assay. Bioorg Med Chem Lett 2011, 21:2192-2197.

26. Abdel-Aal WS, Hassan HY, Aboul-Fadl T, Youssef AF: Pharmacophoric model building for antitubercular activity of the individual Schiff bases of small combinatorial library. Eur J Med Chem 2010, 45:1098-1106.

27. Aboul-Fadl T, Bin-Jubair FAS, Aboul-Wafa V: Schiff bases of indoline-2,3dione (Isatin) derivatives and nalidixic acid carbohydrazide, synthesis, antitubercular activity and pharmacophoric model building. Eur $J$ Med Chem 2010, 45:4578-4586.

28. Aboul-Fadl T, Bin-Jubair FAS: Anti-Tubercular activity of Isatin derivatives. Int J Res Pharm Sci 2010, 1:113-126.

29. Aboul-Fadl T, Abdel-Aziz HA, Kadi A, Ahmad P, Elsaman T, Attwa MW, Darwish IA: Microwave-assisted solution-phase synthesis and DART-mass spectrometric monitoring of combinatorial library of indolin-2,3-dione Schiff bases with potential antimycobacterial activity. Molecules 2011, 16:5194-5206.

30. Aboul-Fadl T, Abdel-Aziz HA, Abdel-Hamid MK, Elsaman T, Thanassi J, Pucci MJ: Schiff bases of indoline-2,3-dione: potential novel inhibitors of mycobacterium tuberculosis (Mtb) DNA gyrase. Molecules 2011, 16:7864-7879.

31. Aboul-Fadl T, Mohammed FA, Hassan EA: Synthesis, antitubercular activity and pharmacokinetic studies of some Schiff bases derived from 1alkylisatin and isonicotinic acid hydrazide (INH). Arch Pharm Re 2003, 26:778-784.

32. Aboul-Fadl T, Radwan AA, Abdel-Aziz HA, Baseeruddin M, Attia MI, Kadi A: Novel Schiff bases of indoline-2,3-dione and nalidixic acid hydrazide: synthesis, in vitro antimycobacterial and in silico mycobacterium tuberculosis (mtb) DNA gyrase inhibitory activity. Dig J Nanomater Bios 2012, 7:327-336.

33. Wolber G, Langer T: LigandScout: 3-D pharmacophores derived from protein-bound ligands and their use as virtual screening filters. J Chem Inf Model 2005, 45:160-169.

34. Lang PT, Brozell SR, Mukherjee S, Pettersen ET, Meng EC, Thomas V, Rizzo RC, Case DA, James TL, Kuntz ID: Dock 6: combining technique to model RNA-small molecule complexes. RNA 2009, 15:1219-1230.

35. Blair JA, Rauh D, Kung C, Yun C-H, Fan Q-W, Rode H, Zhang C, Jeck M, Weiss WA, Shokat KM: Structure-guided development of affinity probes for tyrosine kinases using chemical genetics. Nat Chem Biol 2007, 3:229-238

36. Koch A, Tamez P, Pezzuto J, Soejarto D: Evaluation of plants used for antimalarial treatment by the Massai of Kenya. J Ethnopharmacol 2005, 101:95-99.

37. Badisa RB, Lambert AT, Ikediobi CO, Walker EH: Selective anticancer activity of pure licamichauxiioic-B acid in culture cell lines. Pharmaceut Biol 2006, 44:141-145.

38. Friederike $\mathrm{S}$, Sven $\mathrm{L}$, Thomas $\mathrm{H}$, Karsten $\mathrm{S}$, Philip LF, Hans-dieter $\mathrm{H}$ : Pharmacophore definition and three-dimensional quantitative structure-activity relationship study on structurally diverse prostacyclin receptor agonists. Mol Pharmacol 2002, 62:1103-1111.

39. John S, Thangapandian S, Sakkiah S, Lee KW: Potent bace-1 inhibitor design using pharmacophore modeling, in silico screening and molecular docking studies. BMC Bioinform 2011, 12:S28. doi:10.1186/ 1471-2105-12-S1-S28.

doi:10.1186/1752-153X-6-49

Cite this article as: Aboul-Fadl et al: Schiff bases of indoline-2,3-dione (isatin) with potential antiproliferative activity. Chemistry Central Journal 2012 6:49.

Publish with ChemistryCentral and every
scientist can read your work free of charge
"Open access provides opportunities to our
colleagues in other parts of the globe, by allowing
anyone to view the content free of charge."
W. Jeffery Hurst, The Hershey Company.
- available free of charge to the entire scientific community
- peer reviewed and published immediately upon acceptance
- cited in PubMed and archived on PubMed Central
- yours - you keep the copyright
submit your manuscript here:
http://www.chemistrycentral.com/manuscript/

